\title{
Deep Learning Convolutional Neural Networks for Pharmaceutical Tablet Defect Detection
}

Xiangyu Ma ${ }^{1}$, Nada Kittikunakorn ${ }^{1}$, Bradley Sorman ${ }^{2}$, Hanmi Xi ${ }^{3}$, Antong Chen ${ }^{3}$, Mike Marsh ${ }^{4}$, Arthur Mongeau $^{5}$, Nicolas Piché ${ }^{5}$, Robert Williams III ${ }^{1}$ and Daniel Skomski ${ }^{6}$

${ }^{1}$ The University of Texas at Austin, Austin, Texas, United States, ${ }^{2}$ ExecuPharm, King of Prussia, Pennsylvania, United States, ${ }^{3}$ MRL, Merck \& Co., Inc., West Point, Pennsylvania, United States, ${ }^{4}$ Object Research Systems, Denver, Colorado, United States, ${ }^{5}$ Object Research Systems, Montreal, Quebec, Canada, ${ }^{6}$ Merck \& Co., Inc., Rahway, New Jersey, United States

\section{INTRODUCTION}

Tableting has been widely used in the pharmaceutical industry because of its high throughput and low costs. Recently, internal tablet cracks have been identified as a major risk during the large-scale manufacture of pharmaceutical tablets, which may lead to tablet cracking, capping, and delamination. ${ }^{2}$ These serious problems can result in batch failure and subsequently cause timeline delay, product quality concern, and high financial costs. ${ }^{3}$ Unfortunately, those internal tablet cracks may be overlooked by conventional tests performed routinely during the manufacturing, such as visual appearance inspection. Researchers have demonstrated that X-ray computed tomography (XRCT) is well suited for the detection of these internal tablet cracks. ${ }^{2}$

XRCT normally generates thousands of images, and manual analysis of XRCT data is very difficult to be practically performed every day in the pharmaceutical industry. The manual analysis of all these images is laborious, time-consuming, semi-quantitative, and subjective. ${ }^{2}$ This inability to accurately and quickly identify the internal tablet cracks has hindered the efforts of implementing XRCT to address manufacturing problems of pharmaceutical tablets for better product quality. ${ }^{4}$

In recent years, deep learning convolutional neural networks (CNNs) has gained popularity in various fields, including the pharmaceutical industry. ${ }^{5}$ Due to the development of advanced algorithms, the exponential growth of digitized data, and the development of high computing power hardware, in some cases, deep learning CNNs are capable of matching or even outperforming humans. ${ }^{6}$

In this study, we developed an XRCT analysis program by incorporating deep learning CNNs for internal tablet crack detection. This program can dramatically improve the throughput and accuracy of the internal tablet crack analysis, and most importantly, we demonstrate the high adaptability of this program towards different products with the use of a small amount of training data.

\section{METHODS}

\section{Tablet Preparation}

Two compression forces, $30 \mathrm{kN}$ and $15 \mathrm{kN}$, were used to prepare mannitol-based tablets with internal cracks and mannitol-based tablets without internal cracks, respectively. Ten batches of different ratios of crack/no crack tablets were prepared and placed in four different bottles made of high-density polyethylene (HDPE).

\section{X-Ray Micro-computed Tomography (XRCT)}

All XRCT images of those ten batches of tablets were collected on a SkyScan 1275 micro-CT (Bruker Corporation, Manning Park, MA) at MRL, Merck \& Co., Inc. (Rahway, NJ, USA). Each data set consists 
of 1536 slices with a voxel size of $35 \mu \mathrm{m}$. NRecon reconstruction software (Bruker Corporation, Manning Park, MA) was used to reconstruct all images.

\section{The Architecture of Deep Learning Analysis Program}

The CNN based deep learning analysis program was developed on the DragonFly 4.1 platform licensed by Object Research Systems Inc. (Quebec, Canada). Briefly, this analysis program consists of three independent modules that can be executed automatically:

Module 1: A trained deep learning neural network (UNet A) was used to segment the tablets from the bottle in which they reside;

Module 2: Watershed transformation algorithms were implemented to identify and distinguish each individual tablet from a bulk of tablets;

Module 3: Finally, and most importantly, a second trained deep learning neural network (UNet B) was utilized to identify internal cracks in each tablet and subsequently perform quantitative crack analysis.

\section{RESULTS}

\section{Module 1 \& 2: Tablets-Bottle Segmentation and Individual Tablet Identification}

In this study, the performance of UNet A for the tablets-bottle segmentation was tested and compared to a manual thresholding approach on different bottles. As shown in Fig 1a, UNet A achieved accurate tablets-bottle segmentation compared to the manual thresholding method in which some parts of the bottle were mistakenly segmented as tablets. This accurate segmentation using UNet A is necessary for Module 2 to correctly identify and distinguish individual tablets for further analyses. In Module 2, as shown in Fig. 1b, CNN based deep learning segmentation of bulk tablets resulted in more accurate identification of each tablet compared to the manual thresholding approach. This accuracy achieved in Module 2 ensures correct and precise quantitative analyses of the internal tablet cracks in Module 3.

\section{Module 3: Internal Tablet Crack Analysis}

In Module 3, the accuracy of UNet B of identifying internal tablet cracks was tested on the mannitol-based tablets. As shown in Fig 2a, UNet B exhibited unparalleled performance on detecting internal tablet fractures compared to the manual thresholding approach, which resulted in an extremely inaccurate segmentation of fractures. The analysis program was tested on seven batches with different ratios of crackno crack tablets, and an average accuracy of $94 \%$ was achieved (Fig. 2b). Also, the CNNs based deep learning analysis program is able to execute without user intervention and to process up to 55 tablets at once. It is worth noting that UNet B implemented in this study was trained using only two mannitol-based tablets, taking only 1-2 hours to finish the training process. Overall, the CNNs based deep learning analyzing program showed superior accuracy and flexibility compared to the manual thresholding approach.

\section{CONCLUSION}

In this work, we demonstrate the performance of CNNs based deep learning analysis for analyzing XRCT images and detecting internal tablet fractures. We achieve an average accuracy of $94 \%$ for seven batches of 39-55 tablets. More importantly, the analysis program can be rapidly adapted for different products, 
such as different bottles and tablets. We demonstrate that the CNNs based deep learning approach is suitable for a large-scale manufacturing inspection, which can significantly reduce the time cost and financial cost.

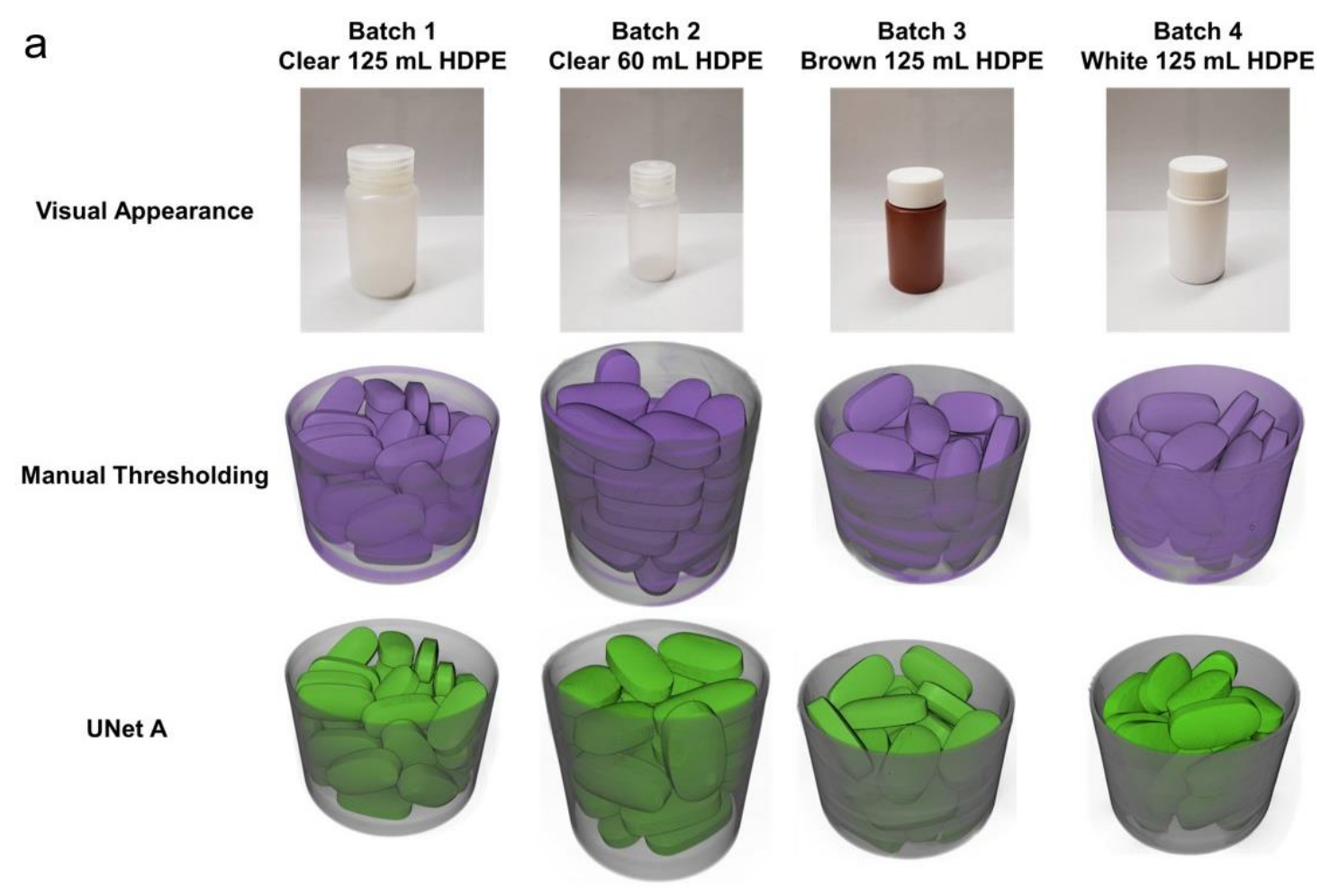

b

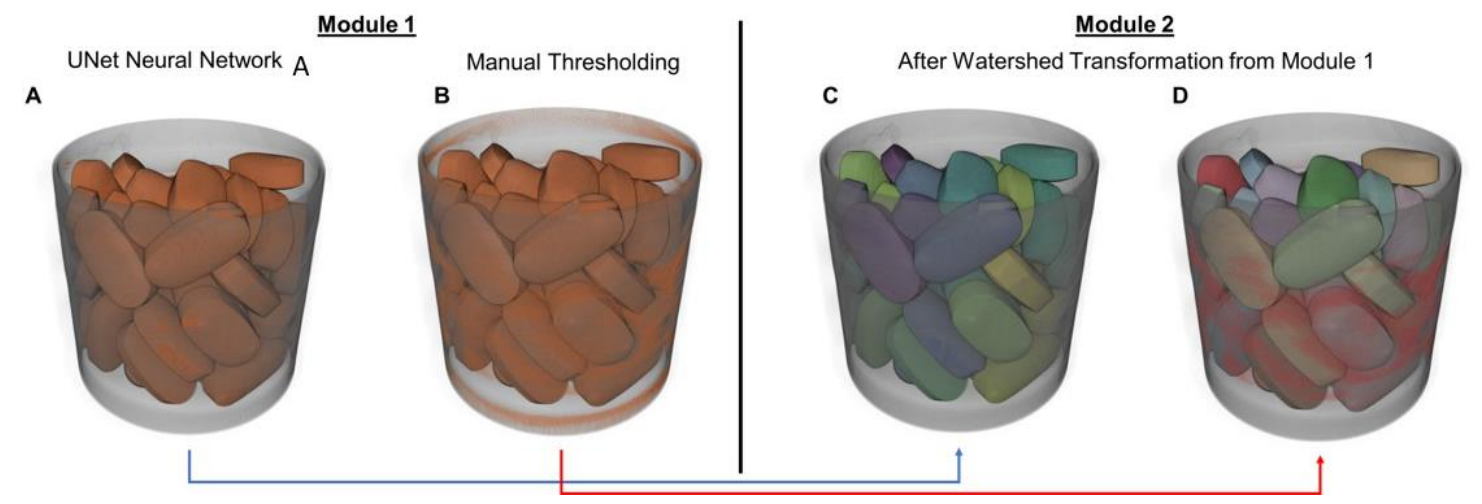

Figure 1. Accurate segmentation of tablets/bottle and tablet/tablets in Module 1 \& 2. (Adapted with permission1) 
a Internal Tablet Cracks

Internal Tablet Cracks Manual Thresholding
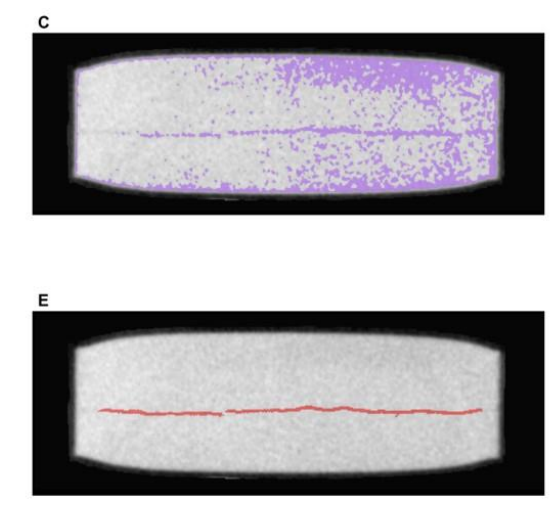
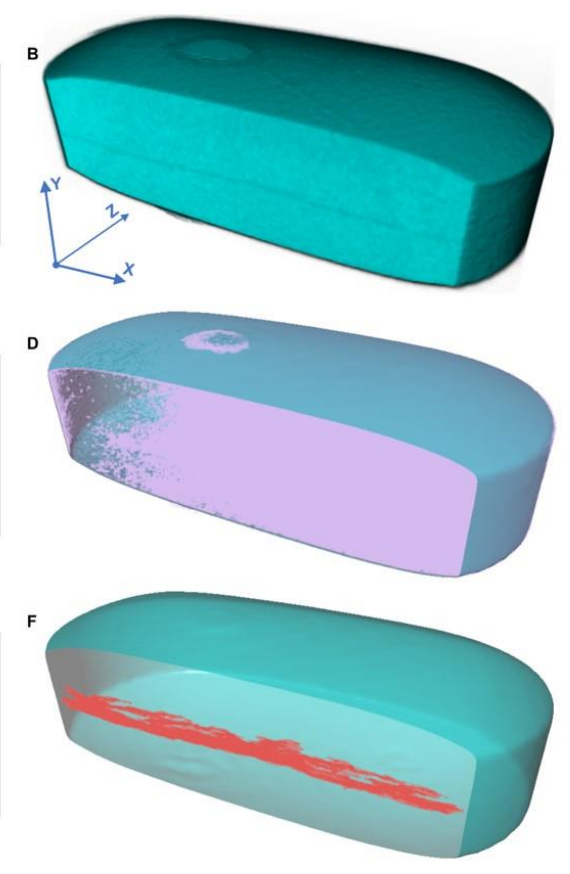

b

\begin{tabular}{|c|c|c|c|c|c|c|c|}
\hline Batch & $\begin{array}{c}\text { Total } \\
\text { Tablets }\end{array}$ & $\begin{array}{l}\text { Tablets with Major } \\
\text { Cracks }\end{array}$ & $\begin{array}{c}\text { Tablets with Minor } \\
\text { Cracks }\end{array}$ & $\begin{array}{c}\text { Tablets with no } \\
\text { Cracks }\end{array}$ & $\begin{array}{c}\text { False } \\
\text { Positives }\end{array}$ & $\begin{array}{c}\text { False } \\
\text { Negatives }\end{array}$ & Accuracy \\
\hline 1 & 39 & 39 & 0 & 0 & 0 & 1 & $97 \%$ \\
\hline 5 & 54 & 1 & 1 & 52 & 2 & 0 & $96 \%$ \\
\hline 6 & 54 & 2 & 1 & 51 & 6 & 0 & $89 \%$ \\
\hline 7 & 55 & 3 & 1 & 51 & 4 & 0 & $93 \%$ \\
\hline 8 & 53 & 5 & 4 & 44 & 5 & 0 & $91 \%$ \\
\hline 9 & 53 & 15 & 3 & 35 & 2 & 0 & $96 \%$ \\
\hline 10 & 54 & 15 & 3 & 36 & 3 & 0 & $94 \%$ \\
\hline \multicolumn{7}{|c|}{ Average } & $94 \%$ \\
\hline \multicolumn{7}{|c|}{ Standard Deviation } & $3 \%$ \\
\hline
\end{tabular}

Figure 2. Accurate internal tablet cracks analysis for seven batches. (Adapted with permission1)

\section{References}

1. Ma X, Kittikunakorn N, Sorman B, Xi H, Chen A, Marsh M, Mongeau A, Piché N, Williams III RO, Skomski D. Application of Deep Learning Convolutional Neural Networks for Internal Tablet Defect Detection: High Accuracy, Throughput, and Adaptability. J Pharm Sci. 2020.

2. Yost E, Chalus P, Zhang S, Peter S, Narang AS. Quantitative X-Ray Microcomputed Tomography Assessment of Internal Tablet Defects. J Pharm Sci. 2019; 108(5):1818-1830.

3. Sabri AH, Hallam CN, Baker NA, Murphy DS, Gabbott IP. Understanding tablet defects in commercial manufacture and transfer. J Drug Deliv Sci Technol. 2018; 46:1-6.

4. Tabernik D, Šela S, Skvarč J, Skočaj D. Segmentation-based deep-learning approach for surface-defect detection. J Intell Manuf. 2019.

5. Calderon CP, Daniels AL, Randolph TW. Deep Convolutional Neural Network Analysis of Flow Imaging Microscopy Data to Classify Subvisible Particles in Protein Formulations. J Pharm Sci. 2018; 107(4):999-1008.

6. Lecun Y, Bengio Y, Hinton G. Deep learning. Nature. 2015; 521(7553):436-444. 